\begin{tabular}{|c|c|}
\hline $\begin{array}{c}\text { 2. To: (Receiving Organization) } \\
\text { Distribution }\end{array}$ & $\begin{array}{l}\text { 3. From: (Originating Organization) } \\
\text { Data Assessment and } \\
\text { Interpretation }\end{array}$ \\
\hline $\begin{array}{l}\text { 5. Proj./Prog./Dept./Div.: } \\
\text { Waste Retrieval Sluicing } \\
\text { System/Waste } \\
\text { Management/DA\&I/Process } \\
\text { Engineering }\end{array}$ & $\begin{array}{l}\text { 6. Design Author ity/ Design Agent/Cog. } \\
\text { Engr.: } \\
\text { Leela M. Sasaki }\end{array}$ \\
\hline
\end{tabular}

11. Receiver Remarks: 11A. Design Baseline Document? [] Yes [X] No For release.

9. Equip./Component No.: $N / A$

10. System/Bldg./Facility: $N / A$

12. Major Assm. Dwg. No.: $N / A$

13. Permit/Permit Application No.: N/A

14. Required Response Date: $03 / 15 / 99$

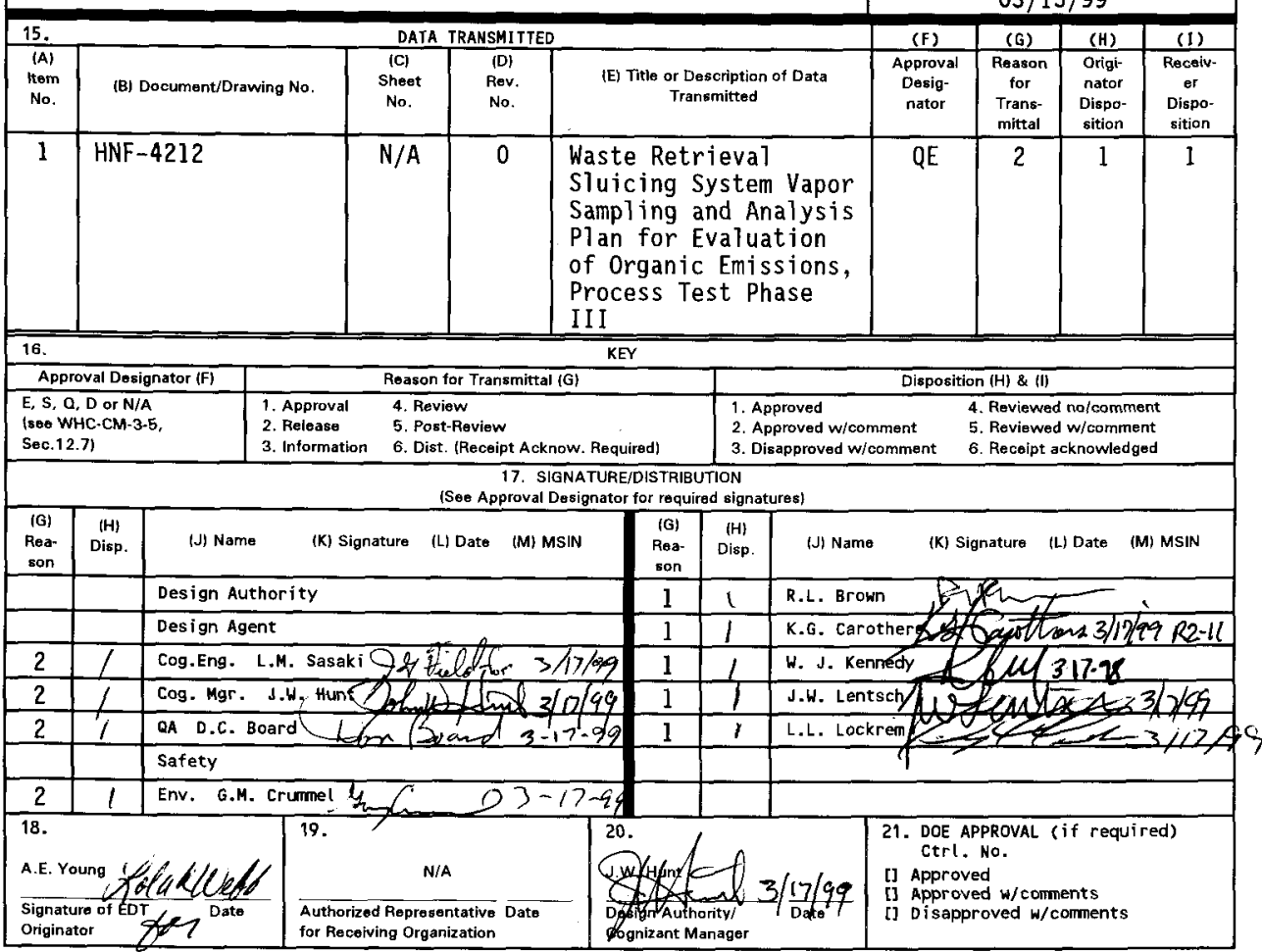

BD-7400-172-2(05/96) GEF097 


\title{
Waste Retrieval Sluicing System Vapor Sampling and Analysis Plan for Evaluation of Organic Emissions, Process Test Phase III
}

\author{
Leela M. Sasaki \\ Lockheed Martin Hanford, Corp., Richland, WA 99352 \\ U.S. Department of Energy Contract DE-AC06-96RL13200 \\ EDT/ECN : EDT-611456 \\ Org Code: 74B20 \\ B\&R Code: EW 3120074 \\ UC: 2070 \\ CACN/COA: $101982 /$ EF00 \\ Total Pages: 32
}

Key Words: Waste Retrieval Sluicing System, WRSS, Vapor, Sampling, Analysis, Plan, Retrieval Project, Characterization, Retrieval, Evaluation, Organic Emissions, Organic, Emission, Process Test, Phase III

Abstract: $\mathrm{N} / \mathrm{A}$

TRADEMARK OISCLAIMER. Reference herein to any specific conmercial product, process, or service by trade name, trademark, manufacturer, or otherwise, does not necessarily constitute or imply its endorsement, recommendation, or favoring by the United States Government or any agency thereof or its contractors or subcontractors.

Printed in the United States of America. To obtain copies of this document, contact: Document Control Services, P.O. Box 950, Mailstop H6-08, Richland WA 99352, Phone (50́9) 372-2420;

Fax (509) 376-4989.
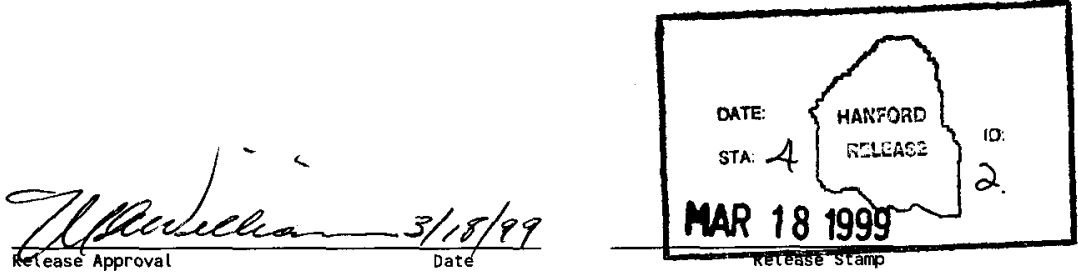

\section{Approved for Public Release}




\section{Waste Retrieval Sluicing System Vapor Sampling and Analysis Plan for Evaluation of Organic Emissions, Process Test Phase III}

L. M. Sasaki

J. A. Johnston

Lockheed Martin Hanford Corporation

Prepared for the U.S. Department of Energy

Office of Environmental Restoration and Waste Management 


\section{TABLE OF CONTENTS}

1.0 SAMPLING AND ANALYSIS OBJECTIVES $\ldots \ldots \ldots \ldots \ldots \ldots \ldots \ldots \ldots$

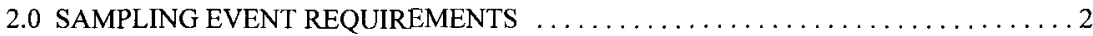

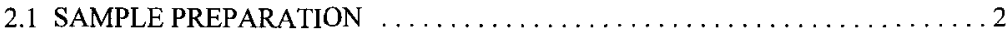

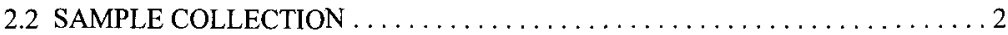

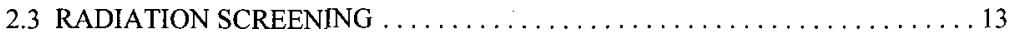

3.0 LABORATORY ANALYSIS REQUIREMENTS $\ldots \ldots \ldots \ldots \ldots \ldots \ldots \ldots \ldots \ldots \ldots$

4.0 QUALITY ASSURANCE AND QUALITY CONTROL $\ldots \ldots \ldots \ldots \ldots \ldots \ldots \ldots 20$

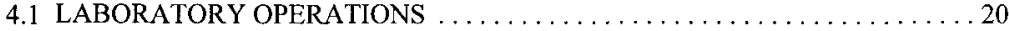

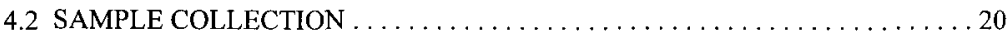

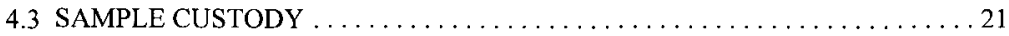

5.0 EXCEPTIONS, CLARIFICATIONS, AND ASSUMPTIONS $\ldots \ldots \ldots \ldots \ldots \ldots 22$

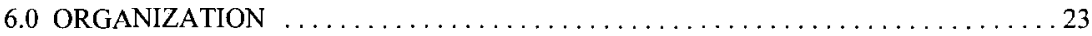

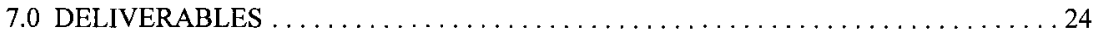

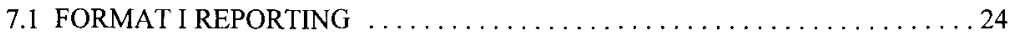

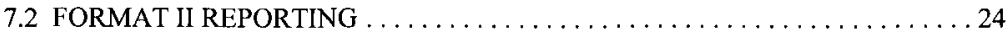

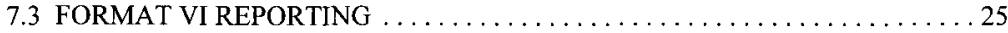

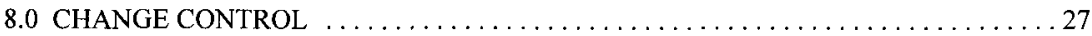

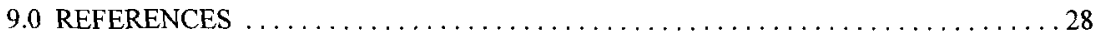

\section{LIST OF TABLES}

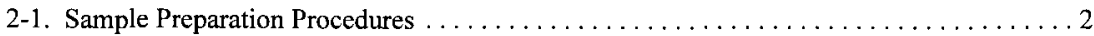

2-2. List of Samples and Activities for 296-C-006 Stack ................... 5

2-3. List of Samples and Activities for Ambient Air Sampling in C Tank Farm . . . . . . . . 11

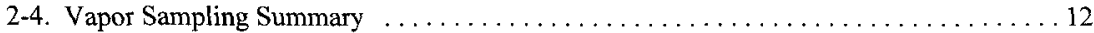

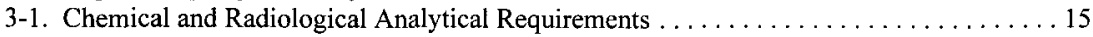

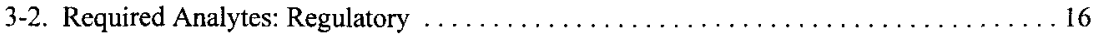

3-3. Required Analytes: Program Requested Analytes/Selected Compounds Observed in Tank 241-C-106 Vapor or Waste Samples . . . . . . . . . . . . . . . . . . . . . 17

3-4. Required Analytes: Target Analytes . . . . . . . . . . . . . . . . . . . 18

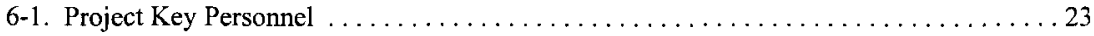

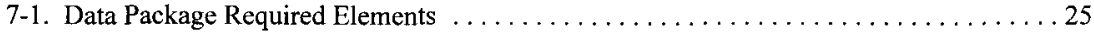


HNF-4212, Rev. 0

\section{LIST OF ABBREVIATIONS}

$\begin{array}{ll}\text { CAS } & \text { Chemical Abstracts Service } \\ \text { CPO } & \text { Characterization Project Operations } \\ \text { DBP } & \text { dibutyl phosphate } \\ \text { FID } & \text { flame ionization detector } \\ \text { GC/MS } & \text { gas chromatography/mass spectrometry } \\ \text { GC/TCD } & \text { gas chromatography/thermal conductivity detector } \\ \text { HASQARD } & \text { Hanford Analytical Services Quality Assurance Requirements Document } \\ \text { IC } & \text { ion chromatography } \\ \text { IDLH } & \text { immediately dangerous to life and health } \\ \text { LFL } & \text { lower flammability limit } \\ \text { LMHC } & \text { Lockheed Martin Hanford Corporation } \\ \text { N/A } & \text { not applicable or not available } \\ \text { NEVS } & \text { non-electrical sampling system } \\ \text { NHC } & \text { Numatec Hanford Corporation } \\ \text { ppmV } & \text { parts per million by volume } \\ \text { PUF } & \text { polyurethane foam } \\ \text { QA } & \text { quality assurance } \\ \text { QC } & \text { quality control } \\ \text { SAS } & \text { Special Analytical Support } \\ \text { STT } & \text { sorbent tube train } \\ \text { TBP } & \text { tributyl phosphate } \\ \text { TOC } & \text { total organic carbon } \\ \text { TST } & \text { triple sorbent trap } \\ \text { TWRS } & \text { Tank Waste Remediation System } \\ \text { VOC } & \text { Volatile Organic Compounds } \\ \text { WSCF } & \text { Waste Sampling and Characterization Facility } \\ \end{array}$




\subsection{SAMPLING AND ANALYSIS OBJECTIVES}

This sampling and analysis plan identifies characterization objectives pertaining to sample collection, laboratory analytical evaluation, and reporting requirements for vapor samples obtained to address vapor issues related to the siuicing of tank 241-C-106. Sampling will be performed in accordance with Waste Retrieval Sluicing System Emissions Collection Phase III (Jones 1999) and Process Test Plan Phase III, Waste Retrieval Sluicing System Emissions Collection (Powers 1999). Analytical requirements include those specified in Request for Ecology Concurrence on Draft Strategy/Path Forward to Address Concerns Regarding Organic Emissions from C-106 Sluicing Activities (Peterson 1998).

The Waste Retrieval Sluicing System was installed to retrieve and transfer high-heat sludge from tank 241-C-106 to tank 241-AY-102, which is designed for high-heat waste storage. During initial sluicing of tank 241-C-106 in November 1998, operations were halted due to detection of unexpected high volatile organic compounds in emissions that exceeded regulatory permit limits. Several workers also reported smelling sharp odors and throat irritation. Vapor grab samples from the 296-C-006 ventilation system were taken as soon as possible after detection; the analyses indicated that volatile and semi-volatile organic compounds were present.

In December 1998, a process test (phase I) was conducted in which the pumps in tanks 241-C106 and 241-AY-102 were operated and vapor samples obtained to determine constituents that may be present during active sluicing of tank 241-C-106. The process test was suspended when a jumper leak was detected.

On March 7, 1999, phase II of the process test was performed; the sluicing system was operated for approximately 7 hours and was ended using the controlled shutdown method when the allowable amount of solids were transferred to 241-AY-102. The phase II test was successful, however, further testing is required to obtain vapor samples at higher emission levels and to demonstrate control of the VOC below acceptable limits during the different modes of sluicing.

A goal of phase III of the process test will be to obtain representative vapor samples at higher concentrations than those achieved in phase I and phase II of the process test. During phase III of the process test, vapor samples will be obtained from the 296-C-006 ventilation system stack. Ambient air samples will also be obtained at two locations within 241-C Tank Farm. Results will be used to address the following:

- Provide gas composition data that can be used to determine personnel protective measures,

- Provide gas composition data that can be used for control equipment selection and design, if required,

- Substantiate that ammonia and organic emissions are below regulatory thresholds during the test.

The following sections provide the general methodology and procedures to be used in the preparation, retrieval, transport, analysis, and reporting of results from vapor samples retrieved during the process test. 
HNF-4212, Rev. 0

\subsection{SAMPLING EVENT REQUIREMENTS}

The waste retrieval sluicing system will be operated in accordance with the process test plan and process test procedure (Powers 1999, Jones 1999) to raise the volatile organic compound (VOC) concentration in the 296-C-006 ventilation stack to levels above those reached in phase I and phase II of the process test. It is anticipated that stack VOC levels of approximately $300-400$ parts per million by volume (ppmv) will be reached. Based on this information, waste agitation will be controlled at approximately 325 ppmv. The pumps will be tripped at $400 \mathrm{ppmv}$. Emergency evacuation will be conducted at $500 \mathrm{ppmv}$. The effects of the ventilation recirculation heater may be tested. Total sluicing duration for the performance of this VOC process testing is limited to approximately 13 hours. Vapor samples will be taken prior to and during pump operation.

\subsection{SAMPLE PREPARATION}

SUMMA ${ }^{1}$ canister samples, triple sorbent trap (TST) samples, sorbent tube train (STT) samples, and polyurethane foam (PUF) samples will be collected and sent to the laboratory for analysis; field and trip blanks will accompany the samples. The analytical laboratory for this sampling event is Numatec Hanford Corporation (NHC) Special Analytical Support (SAS). Particulate filter samples will be collected for radiation screening of the samples. SAS shall prepare the SUMMA canisters, TSTs, STTs, and PUFs for sample collection. SAS shall also prepare the particulate filter assemblies for use at the 296-C-006 stack; each particulate filter assembly shall contain two particulate filters mounted in series. Sample preparation procedures are listed in Table 2-1.

Table 2-1. Sample Preparation Procedures

\begin{tabular}{|l|l|l|}
\hline Sample container & Organization & Preparation Procedure \\
\hline SUMMA canister & SAS & LO-080-406 \\
\hline TST & SAS & LA-549-403 \\
\hline STT & SAS & LA-549-402 \\
\hline PUF & SAS & N/A \\
\hline Particulate filter assembly & SAS & N/A \\
\hline
\end{tabular}

Note:

$\mathrm{N} / \mathrm{A}=$ not applicable

\subsection{SAMPLE COLLECTION}

CPO will be responsible for the collection of all SUMMA canister samples. SAS will be responsible for the collection of all particulate filter, TST, STT, and PUF samples.

\footnotetext{
'SUMMA is a trademark of Molectrics of Ohio.
} 


\section{HNF-4212, Rev. 0}

CPO sampling activities will be performed in accordance with work package ES-99-00090. SAS sampling activities will be performed in accordance with work package 2E-99-00577 and procedure L0-080-400, Vapor Sampling Using the Non-Electrical Vapor Sampling System $(N E V S)$.

Tubing used in the collection of vapor samples must be stainless steel, Teflon ${ }^{2}$, or Teflon-coated and the length of the tubing used should be minimized. No C-flex or tygon tubing should be used upstream of the sample containers.

The samples shall include the following quality control (QC) samples: one each TST, STT, and PUF ambient air field blank; and one each TST, STT, and PUF trip blank. The trip and field blanks are to accompany the vapor samples to the laboratory. For specific information concerning sample and blank handling, custody, and transport, refer to the requirements in Section 4.0 .

CPO and SAS shall record (or calculate) the following information for each sample collected: sample number, start and stop times for the collection of each sample, the VOC reading at the start of the collection of each sample, sample volumes, and any anomalous sampling conditions.

Particulate filter samples shall be shipped by CPO to the Waste Sampling and Characterization Facility (WSCF) for radiation screening analysis. The remaining samples shall be stored by $\mathrm{CPO}$ until radiation screening is completed and the samples can be released for analysis. Upon completion of the radiation screening and release of the samples, the samples shall be transferred by CPO to SAS for analysis.

Tables 2-2 and 2-3 provide the sequence of sampling activities for the 296-C-006 stack and ambient air samples, respectively. The samples are summarized in Table 2-4. SUMMA, TST, STT, and PUF samples will not be collected through particulate filters. The particulate filter samples shall be collected separately, and the filter assembly removed from the sampling port prior to collecting the other samples.

At a minimum, the following samples are required from the 296-C-006 stack:

- $\quad$ One set of samples before the pumps are started (baseline samples)

- Two to three sets of samples after the pumps have started, as the stack VOC (as measured by the flame ionization detector [FID]) is increasing

- Two to three sets of samples during peak or steady state VOC concentrations

To ensure that these samples are obtained, it is recommended that one set of samples be collected from the 296-C-006 stack when the VOC concentration has reached $50 \mathrm{ppmv}$, one set collected when the VOC concentration reaches $200 \mathrm{ppmv}$, a set collected once per hour or at the test director's direction, and a special set of SUMMAs (15L and 35L) during peak/steady state (see Table 2-2). When three sets of samples have been collected at the peak/steady state VOC concentration, no further samples are required from the 296-C-006 stack. Sample collection or

${ }^{2}$ Teflon is a registered trademark of I. E. DuPont De Nemours and Company 


\section{HNF-4212, Rev. 0}

the suspension of sampling activities may also occur at the instruction of the test director. The sampling activities of SAS and CPO shall be coordinated so that, for each set of samples, the SUMMA canisters, TSTs, STTs, and PUFs are obtained at about the same time.

Ambient air samples in the $\mathrm{C}$ tank farm will consist of samples at two locations: 1) near the 296C-006 stack, and 2) downwind of the stack at the fresh air boundary. Samples shall be obtained at these locations before the sluicing pumps are started and again when the VOC concentrations in the stack reach their peak or steady state concentrations.

Any decisions, observations, or deviations affecting this SAP shall be documented in controlled notebooks/or work packages and justified in the deliverable report. 
HNF-4212, Rev. 0

Table 2-2. List of Samples and Activities for 296-C-006 Stack (6 sheets).

\begin{tabular}{|c|c|c|c|c|}
\hline $\begin{array}{l}\text { Sample } \\
\text { Code }\end{array}$ & Sample/Activity Description & $\begin{array}{l}\text { Sampler Position } \\
\text { During Collection }\end{array}$ & $\begin{array}{l}\text { Sample } \\
\text { Volume }\end{array}$ & $\begin{array}{c}\text { Sample } \\
\text { Duration } \\
\text { or Flow } \\
\text { Rate }\end{array}$ \\
\hline-- & Perform leak test of NEVS & N/A & N/A & $\mathrm{N} / \mathrm{A}$ \\
\hline \multicolumn{5}{|c|}{ Baseline samples } \\
\hline 401 & $\begin{array}{l}\text { Collect particulate filter sample before pump } \\
\text { is started }\end{array}$ & 296-C-006 Stack & $1 \mathrm{~L}$ & $\begin{array}{l}\leq 500 \\
\mathrm{~mL} / \mathrm{min}\end{array}$ \\
\hline 402 & $\begin{array}{l}\text { Collect baseline SUMMA canister before } \\
\text { pump is started }\end{array}$ & 296-C-006 Stack & $6 \mathrm{~L}$ & $2 \mathrm{~min}$ \\
\hline 403 & Collect baseline TST before pump is started & 296-C-006 Stack & $0.5 \mathrm{~L}$ & $\begin{array}{l}\leq 500 \\
\mathrm{~mL} / \mathrm{min}\end{array}$ \\
\hline 404 & Collect baseline STT before pump is started & 296-C-006 Stack & $6 \mathrm{~L}$ & $\begin{array}{l}\leq 500 \\
\mathrm{~mL} / \mathrm{min}\end{array}$ \\
\hline 405 & $\begin{array}{l}\text { Collect baseline PUF trap before pump is } \\
\text { started }\end{array}$ & 296-C-006 Stack & $25 \mathrm{~L}$ & $\leq 5 \mathrm{~L} / \mathrm{min}$ \\
\hline \multicolumn{5}{|c|}{ Sampling during sluicing operations } \\
\hline \multicolumn{5}{|c|}{ Collect sample set $\# 1$ when voc rises to 50 ppmv. } \\
\hline 406 & Collect particulate filter sample & 296-C-006 Stack & $1 \mathrm{~L}$ & $\begin{array}{l}\leq 500 \\
\mathrm{~mL} / \mathrm{min}\end{array}$ \\
\hline 407 & Collect SUMMA canister & 296-C-006 Stack & $6 \mathrm{~L}$ & $2 \mathrm{~min}$ \\
\hline 408 & Collect STT & 296-C-006 Stack & $6 \mathrm{~L}$ & $\begin{array}{l}\leq 500 \\
\mathrm{~mL} / \mathrm{min}\end{array}$ \\
\hline 409 & Collect TST & 296-C-006 Stack & $0.1 \mathrm{~L}$ & $\begin{array}{l}\leq 500 \\
\mathrm{~mL} / \mathrm{min}\end{array}$ \\
\hline 410 & Collect PUF trap & 296-C-006 Stack & $25 \mathrm{~L}$ & $\leq 5 \mathrm{~L} / \mathrm{min}$ \\
\hline \multicolumn{5}{|c|}{ Collect sample set $\# 2$ when VOC rises to 200 ppmv. } \\
\hline 411 & Collect particulate filter sample & 296-C-006 Stack & $1 \mathrm{~L}$ & $\begin{array}{l}\leq 500 \\
\mathrm{~mL} / \min \end{array}$ \\
\hline 412 & Collect SUMMA canister & 296-C-006 Stack & $6 \mathrm{~L}$ & $2 \mathrm{~min}$ \\
\hline 413 & Collect STT & 296-C-006 Stack & $6 \mathrm{~L}$ & $\begin{array}{l}\leq 500 \\
\mathrm{~mL} / \mathrm{min}\end{array}$ \\
\hline 414 & Collect TST & 296-C-006 Stack & $0.1 \mathrm{~L}$ & $\begin{array}{l}\leq 500 \\
\mathrm{~mL} / \mathrm{min}\end{array}$ \\
\hline 415 & Collect PUF trap & 296-C-006 Stack & $25 \mathrm{~L}$ & $\leq 5 \mathrm{~L} / \mathrm{min}$ \\
\hline
\end{tabular}


HNF-4212, Rev. 0

Table 2-2. List of Samples and Activities for 296-C-006 Stack (6 sheets).

\begin{tabular}{|c|c|c|c|c|}
\hline $\begin{array}{l}\text { Sample } \\
\text { Code }\end{array}$ & Sample/Activity Description & $\begin{array}{l}\text { Sampler Position } \\
\text { During Collection }\end{array}$ & $\begin{array}{l}\text { Sample } \\
\text { Volume }\end{array}$ & $\begin{array}{l}\text { Sample } \\
\text { Duration } \\
\text { or Flow } \\
\text { Rate }\end{array}$ \\
\hline \multicolumn{5}{|c|}{ Collect sample set $\# 3$ one hour after collection of sample set $\# 2$. } \\
\hline 416 & Collect particulate filter sample & 296-C-006 Stack & $1 \mathrm{~L}$ & $\begin{array}{l}\leq 500 \\
\mathrm{~mL} / \mathrm{min}\end{array}$ \\
\hline 417 & Collect SUMMA canister & 296-C-006 Stack & $6 \mathrm{~L}$ & $2 \mathrm{~min}$ \\
\hline 418 & Collect STT & 296-C-006 Stack & $6 \mathrm{~L}$ & $\begin{array}{l}\leq 500 \\
\mathrm{~mL} / \mathrm{min}\end{array}$ \\
\hline 419 & Collect TST & 296-C-006 Stack & $0.1 \mathrm{~L}$ & $\begin{array}{l}\leq 500 \\
\mathrm{~mL} / \mathrm{min}\end{array}$ \\
\hline 420 & Collect PUF trap & 296-C-006 Stack & $25 \mathrm{~L}$ & $\preceq 5 \mathrm{~L} / \mathrm{min}$ \\
\hline \multicolumn{5}{|c|}{ Collect sample set \#4 one hour after collection of sample set \#3. } \\
\hline 421 & Collect particulate filter sample & 296-C-006 Stack & $1 \mathrm{~L}$ & $\begin{array}{l}\leq 500 \\
\mathrm{~mL} / \mathrm{min}\end{array}$ \\
\hline 422 & Collect SUMMA canister & 296-C-006 Stack & $6 \mathrm{~L}$ & $2 \mathrm{~min}$ \\
\hline 423 & Collect STT & 296-C-006 Stack & $6 \mathrm{~L}$ & $\begin{array}{l}\leq 500 \\
\mathrm{~mL} / \mathrm{min}\end{array}$ \\
\hline 424 & Collect TST & 296-C-006 Stack & $0.1 \mathrm{~L}$ & $\begin{array}{l}\leq 500 \\
\mathrm{~mL} / \mathrm{min}\end{array}$ \\
\hline 425 & Collect PUF trap & 296-C-006 Stack & $25 \mathrm{~L}$ & $\preceq 5 \mathrm{~L} / \mathrm{min}$ \\
\hline \multicolumn{5}{|c|}{ Collect sample set $\$ 5$ one hour after collection of sample set $\# 4$. } \\
\hline 426 & Collect particulate filter sample & 296-C-006 Stack & $1 \mathrm{~L}$ & $\begin{array}{l}\leq 500 \\
\mathrm{~mL} / \mathrm{min}\end{array}$ \\
\hline 427 & Collect SUMMA canister & 296-C-006 Stack & $6 \mathrm{~L}$ & $2 \mathrm{~min}$ \\
\hline 428 & Collect STT & 296-C-006 Stack & $6 \mathrm{~L}$ & $\begin{array}{l}\leq 500 \\
\mathrm{~mL} / \mathrm{min}\end{array}$ \\
\hline 429 & Collect TST & 296-C-006 Stack & $0.1 \mathrm{~L}$ & $\begin{array}{l}\leq 500 \\
\mathrm{~mL} / \mathrm{min}\end{array}$ \\
\hline 430 & Collect PUF trap & 296-C-006 Stack & $25 \mathrm{~L}$ & $\leq 5 \mathrm{~L} / \mathrm{min}$ \\
\hline
\end{tabular}


HNF-4212, Rev. 0

Table 2-2. List of Samples and Activities for 296-C-006 Stack (6 sheets).

\begin{tabular}{|c|c|c|c|c|}
\hline $\begin{array}{l}\text { Sample } \\
\text { Code }\end{array}$ & Sample/Activity Description & $\begin{array}{l}\text { Sampler Position } \\
\text { During Collection }\end{array}$ & $\begin{array}{l}\text { Sample } \\
\text { Volume }\end{array}$ & $\begin{array}{c}\text { Sample } \\
\text { Duration } \\
\text { or Flow } \\
\text { Rate }\end{array}$ \\
\hline \multicolumn{5}{|c|}{ Collect sample set $\# 6$ one hour after collection of sample set 45 . } \\
\hline 431 & Collect particulate filter sample & 296-C-006 Stack & $1 \mathrm{~L}$ & $\begin{array}{l}\leq 500 \\
\mathrm{~mL} / \mathrm{min}\end{array}$ \\
\hline 432 & Collect SUMMA canister & 296-C-006 Stack & $6 \mathrm{~L}$ & $2 \mathrm{~min}$ \\
\hline 433 & Collect STT & 296-C-006 Stack & $6 \mathrm{~L}$ & $\begin{array}{l}500 \\
\mathrm{~mL} / \mathrm{min}\end{array}$ \\
\hline 434 & Collect TST & 296-C-006 Stack & $0.1 \mathrm{~L}$ & $\begin{array}{l}\leq 500 \\
\mathrm{~mL} / \mathrm{min}\end{array}$ \\
\hline 435 & Collect PUF trap & 296-C-006 Stack & $25 \mathrm{~L}$ & $\preceq 5 \mathrm{~L} / \mathrm{min}$ \\
\hline \multicolumn{5}{|c|}{ Collect sample set $\# 7$ one hour after collection of sample set $\# 6$. } \\
\hline 436 & Collect particulate filter sample & 296-C-006 Stack & $1 \mathrm{~L}$ & $\begin{array}{l}\leq 500 \\
\mathrm{~mL} / \mathrm{min}\end{array}$ \\
\hline 437 & Collect SUMMA canister & 296-C-006 Stack & $6 \mathrm{~L}$ & $2 \min$ \\
\hline 438 & Collect STT & 296-C-006 Stack & $6 \mathrm{~L}$ & $\begin{array}{l}\leq 500 \\
\mathrm{~mL} / \mathrm{min}\end{array}$ \\
\hline 439 & Collect TST & 296-C-006 Stack & $0.1 \mathrm{~L}$ & $\begin{array}{l}\leq 500 \\
\mathrm{~mL} / \mathrm{min}\end{array}$ \\
\hline 440 & Collect PUF trap & 296-C-006 Stack & $25 \mathrm{~L}$ & $\leq 5 \mathrm{~L} / \mathrm{min}$ \\
\hline \multicolumn{5}{|c|}{ Collect sample set $\# 8$ one hour after collection of sample set $\# 7$. } \\
\hline 441 & Collect particulate filter sample & 296-C-006 Stack & $1 \mathrm{~L}$ & $\begin{array}{l}\preceq 500 \\
\mathrm{~mL} / \mathrm{min}\end{array}$ \\
\hline 442 & Collect SUMMA canister & 296-C-006 Stack & $6 \mathrm{~L}$ & $2 \mathrm{~min}$ \\
\hline 443 & Collect STT & 296-C-006 Stack & $6 \mathrm{~L}$ & $\begin{array}{l}\leq 500 \\
\mathrm{~mL} / \mathrm{min}\end{array}$ \\
\hline 444 & Collect TST & 296-C-006 Stack & $0.1 \mathrm{~L}$ & $\begin{array}{l}\leq 500 \\
\mathrm{~mL} / \mathrm{min}\end{array}$ \\
\hline 445 & Collect PUF trap & 296-C-006 Stack & $25 \mathrm{~L}$ & $\leq 5 \mathrm{~L} / \mathrm{min}$ \\
\hline
\end{tabular}


HNF-4212, Rev. 0

Table 2-2. List of Samples and Activities for 296-C-006 Stack (6 sheets).

\begin{tabular}{|c|c|c|c|c|}
\hline $\begin{array}{l}\text { Sample } \\
\text { Code }\end{array}$ & Sample/Activity Description & $\begin{array}{l}\text { Sampler Position } \\
\text { During Collection }\end{array}$ & $\begin{array}{l}\text { Sample } \\
\text { Volume }\end{array}$ & $\begin{array}{l}\text { Sample } \\
\text { Duration } \\
\text { or Flow } \\
\text { Rate }\end{array}$ \\
\hline \multicolumn{5}{|c|}{ Collect sample set $\$ 9$ one hour after collection of sample set $\# 8$} \\
\hline 446 & Collect particulate filter sample & 296-C-006 Stack & $1 \mathrm{~L}$ & $\begin{array}{l}\leq 500 \\
\mathrm{~mL} / \mathrm{min}\end{array}$ \\
\hline 447 & Collect SUMMA canister & 296-C-006 Stack & $6 \mathrm{~L}$ & $2 \min$ \\
\hline 448 & Collect STT & 296-C-006 Stack & $6 \mathrm{~L}$ & $\begin{array}{l}\leq 500 \\
\mathrm{~mL} / \mathrm{min}\end{array}$ \\
\hline 449 & Collect TST & 296-C-006 Stack & $0.1 \mathrm{~L}$ & $\begin{array}{l}\leq 500 \\
\mathrm{~mL} / \mathrm{min}\end{array}$ \\
\hline 450 & Collect PUF trap & 296-C-006 Stack & $25 \mathrm{~L}$ & $\preceq 5 \mathrm{~L} / \mathrm{min}$ \\
\hline \multicolumn{5}{|c|}{ Collect sample set $\# 10$ one hour after collection of sample set $\# 9$} \\
\hline 451 & Collect particulate filter sample & $296 \cdot \mathrm{C}-006$ Stack & $1 \mathrm{~L}$ & $\begin{array}{l}\leq 500 \\
\mathrm{~mL} / \mathrm{min}\end{array}$ \\
\hline 452 & Collect SUMMA canister & 296-C-006 Stack & $6 \mathrm{~L}$ & $2 \mathrm{~min}$ \\
\hline 453 & Collect STT & 296-C-006 Stack & $6 \mathrm{~L}$ & $\begin{array}{l}\leq 500 \\
\mathrm{~mL} / \mathrm{min}\end{array}$ \\
\hline 454 & Collect TST & 296-C-006 Stack & $0.1 \mathrm{~L}$ & $\begin{array}{l}\leq 500 \\
\mathrm{~mL} / \mathrm{min}\end{array}$ \\
\hline 455 & Collect PUF trap & 296-C-006 Stack & $25 \mathrm{~L}$ & $\preceq 5 \mathrm{~L} / \mathrm{min}$ \\
\hline \multicolumn{5}{|c|}{ Collect sample set $\# 11$ one hour after collection of sample set 110 . } \\
\hline 456 & Collect particulate filter sample & 296-C-006 Stack & $1 \mathrm{~L}$ & $\begin{array}{l}\leq 500 \\
\mathrm{~mL} / \mathrm{min}\end{array}$ \\
\hline 457 & Collect SUMMA canister & 296-C-006 Stack & $6 \mathrm{~L}$ & $2 \mathrm{~min}$ \\
\hline 458 & Collect STT & 296-C-006 Stack & $6 \mathrm{~L}$ & $\begin{array}{l}\leq 500 \\
\mathrm{~mL} / \mathrm{min}\end{array}$ \\
\hline 459 & Collect TST & 296-C-006 Stack & $0.1 \mathrm{~L}$ & $\begin{array}{l}\leq 500 \\
\mathrm{~mL} / \mathrm{min}\end{array}$ \\
\hline 460 & Collect PUF trap & 296-C-006 Stack & $25 \mathrm{~L}$ & $\leq 5 \mathrm{~L} / \mathrm{min}$ \\
\hline
\end{tabular}


HNF-4212, Rev. 0

Table 2-2. List of Samples and Activities for 296-C-006 Stack (6 sheets).

\begin{tabular}{|c|c|c|c|c|}
\hline $\begin{array}{l}\text { Sample } \\
\text { Code }\end{array}$ & Sample/Activity Description & $\begin{array}{l}\text { Sampler Position } \\
\text { During Collection }\end{array}$ & $\begin{array}{l}\text { Sample } \\
\text { Volume }\end{array}$ & $\begin{array}{l}\text { Sample } \\
\text { Duration } \\
\text { or Flow } \\
\text { Rate }\end{array}$ \\
\hline \multicolumn{5}{|c|}{ Collect sample set $\#$ i 2 one hour after collection of sample set $\# 11$. } \\
\hline 461 & Collect particulate filter sample & 296-C-006 Stack & $1 \mathrm{~L}$ & $\begin{array}{l}\leq 500 \\
\mathrm{~mL} / \mathrm{min}\end{array}$ \\
\hline 462 & Collect SUMMA canister & 296-C-006 Stack & $6 \mathrm{~L}$ & $2 \mathrm{~min}$ \\
\hline 463 & Collect STT & 296-C-006 Stack & $6 \mathrm{~L}$ & $\begin{array}{l}\leq 500 \\
\mathrm{~mL} / \mathrm{min}\end{array}$ \\
\hline 464 & Collect TST & 296-C-006 Stack & $0.1 \mathrm{~L}$ & $\begin{array}{l}\leq 500 \\
\mathrm{~mL} / \mathrm{min}\end{array}$ \\
\hline 465 & Collect PUF trap & 296-C-006 Stack & $25 \mathrm{~L}$ & $\leq 5 \mathrm{~L} / \mathrm{min}$ \\
\hline \multicolumn{5}{|c|}{ Collect sample set $\# 13$ one hour after collection of sample set $\# 12$. } \\
\hline 466 & Collect particulate filter sample & 296. C-006 Stack & $1 \mathrm{~L}$ & $\begin{array}{l}\leq 500 \\
\mathrm{~mL} / \mathrm{min}\end{array}$ \\
\hline 467 & Collect SUMMA canister & 296-C-006 Stack & $6 \mathrm{~L}$ & $2 \mathrm{~min}$ \\
\hline 468 & Collect STT & 296-C-006 Stack & $6 \mathrm{~L}$ & $\begin{array}{l}₫ 500 \\
\mathrm{~mL} / \mathrm{min}\end{array}$ \\
\hline 469 & Collect TST & 296-C-006 Stack & $0.1 \mathrm{~L}$ & $\begin{array}{l}\leq 500 \\
\mathrm{~mL} / \mathrm{min}\end{array}$ \\
\hline 470 & Collect PUF trap & 296-C-006 Stack & $25 \mathrm{~L}$ & $\leq 5 \mathrm{~L} / \mathrm{min}$ \\
\hline \multicolumn{5}{|c|}{ Collect sample set $\# 14$ one hour after collection of sample set $\# 13$. } \\
\hline 471 & Collect particulate filter sample & 296-C-006 Stack & $1 \mathrm{~L}$ & $\begin{array}{l}\leq 500 \\
\mathrm{~mL} / \mathrm{min}\end{array}$ \\
\hline 472 & Collect SUMMA canister & 296-C-006 Stack & $6 \mathrm{~L}$ & $2 \mathrm{~min}$ \\
\hline 473 & Collect STT & 296-C-006 Stack & $6 \mathrm{~L}$ & $\begin{array}{l}\leq 500 \\
\mathrm{~mL} / \mathrm{min}\end{array}$ \\
\hline 474 & Collect TST & 296-C-006 Stack & $0.1 \mathrm{~L}$ & $\begin{array}{l}\leq 500 \\
\mathrm{~mL} / \mathrm{min}\end{array}$ \\
\hline 475 & Collect PUF trap & 296-C-006 Stack & $25 \mathrm{~L}$ & $\checkmark 5 \mathrm{~L} / \mathrm{min}$ \\
\hline
\end{tabular}


HNF-4212, Rev. 0

Table 2-2. List of Samples and Activities for 296-C-006 Stack (6 sheets).

\begin{tabular}{|c|c|c|c|c|}
\hline $\begin{array}{l}\text { Sample } \\
\text { Code }\end{array}$ & Sample/Activity Description & $\begin{array}{l}\text { Sampler Position } \\
\text { During Collection }\end{array}$ & $\begin{array}{l}\text { Sample } \\
\text { Volume }\end{array}$ & $\begin{array}{l}\text { Sample } \\
\text { Duration } \\
\text { or Flow } \\
\text { Rate }\end{array}$ \\
\hline \multicolumn{5}{|c|}{ Collect sample set $\# 15$ one hour after collection of sample set $\# 14$} \\
\hline 476 & Collect particulate filter sample & 296-C-006 Stack & $1 \mathrm{~L}$ & $\begin{array}{l}\leq 500 \\
\mathrm{~mL} / \mathrm{min}\end{array}$ \\
\hline 477 & Collect SUMMA canister & 296-C-006 Stack & $6 \mathrm{~L}$ & $2 \mathrm{~min}$ \\
\hline 478 & Collect STT & 296-C-006 Stack & $6 \mathrm{~L}$ & $\begin{array}{l}\leq 500 \\
\mathrm{~mL} / \mathrm{min}\end{array}$ \\
\hline 479 & Collect TST & 296-C-006 Stack & $0.1 \mathrm{~L}$ & $\begin{array}{l}\leq 500 \\
\mathrm{~mL} / \mathrm{min}\end{array}$ \\
\hline 480 & Collect PUF trap & 296-C-006 Stack & $25 \mathrm{~L}$ & $\leq 5 \mathrm{~L} / \mathrm{min}$ \\
\hline \multicolumn{5}{|c|}{ Special sample set to be collected during peak/steady state } \\
\hline 481 & Collect SUMMA canister & 296-C-006 Stack & $15 \mathrm{~L}$ & $5 \mathrm{~min}$ \\
\hline 482 & Collect SUMMA canister & 296-C-006 Stack & $35 \mathrm{~L}$ & $10 \mathrm{~min}$ \\
\hline \multicolumn{5}{|c|}{ Blanks } \\
\hline 483 & Store TST Trip Blank & -- & --- & -- \\
\hline 484 & Store STT Trip Blank & -- & -- & -- \\
\hline 485 & Store PUF Trip Blank & -- & -- & --- \\
\hline 486 & Open, close, and store TST Field Blank & $\cdots$ & -- & -- \\
\hline 487 & Open, close, and store STT Field Blank & -- & -- & -- \\
\hline 486 & Open, close, and store PUF Field Blank & -- & -- & -- \\
\hline
\end{tabular}

Note:

'CPO and SAS may adjust sample durations and flow rates as necessary to collect the samples. 
HNF-4212, Rev. 0

Table 2-3. List of Samples and Activities for Ambient Air Sampling in C Tank Farm

\begin{tabular}{|c|c|c|c|c|}
\hline $\begin{array}{l}\text { Sample } \\
\text { Code }\end{array}$ & Sample/Activity Description & $\begin{array}{l}\text { Sampler Position } \\
\text { During Collection }\end{array}$ & $\begin{array}{l}\text { Sample } \\
\text { Volume }\end{array}$ & $\begin{array}{l}\text { Sample } \\
\text { Duration } \\
\text { or Rate }\end{array}$ \\
\hline \multicolumn{5}{|c|}{ Baseline samples } \\
\hline 401 & $\begin{array}{l}\text { Collect baseline SUMMA before pump is } \\
\text { started. }\end{array}$ & near 296-C-006 stack & $6 \mathrm{~L}$ & $1 \mathrm{~min}$ \\
\hline 402 & Collect baseline TST before pump is started & near 296-C-006 stack & $1 \mathrm{~L}$ & $\begin{array}{l}\preceq 500 \\
\mathrm{~mL} / \mathrm{min}\end{array}$ \\
\hline 403 & Collect baseline STT before pump is started & near 296-C-006 stack & $6 \mathrm{~L}$ & $\begin{array}{l}\leq 500 \\
\mathrm{~mL} / \mathrm{min}\end{array}$ \\
\hline 404 & Collect baseline PUF before pump is started & near 296-C-006 stack & $25 \mathrm{~L}$ & $\leq 5 \mathrm{~L} / \mathrm{min}$ \\
\hline 405 & $\begin{array}{l}\text { Collect baseline SUMMA before pump is } \\
\text { started }\end{array}$ & $\begin{array}{l}\text { downwind at the } \\
\text { fresh air boundary }\end{array}$ & $6 \mathrm{~L}$ & $1 \mathrm{~min}$ \\
\hline \multicolumn{5}{|c|}{ Samples at peak/steady state VOC concentrations } \\
\hline 406 & $\begin{array}{l}\text { Collect SUMMA when stack VOC } \\
\text { concentration has reached peak/steady state }\end{array}$ & near 296-C-006 stack & $6 \mathrm{~L}$ & $1 \mathrm{~min}$ \\
\hline 407 & $\begin{array}{l}\text { Collect TST when stack VOC concentration } \\
\text { has reached peak/steady state }\end{array}$ & near 296-C-006 stack & $1 \mathrm{~L}$ & $\begin{array}{l}\leq 500 \\
\mathrm{~mL} / \mathrm{min}\end{array}$ \\
\hline 408 & $\begin{array}{l}\text { Collect STT when stack VOC concentration } \\
\text { has reached peak/steady state }\end{array}$ & near 296-C-006 stack & $6 \mathrm{~L}$ & $\begin{array}{l}\leq 500 \\
\mathrm{~mL} / \mathrm{min}\end{array}$ \\
\hline 409 & $\begin{array}{l}\text { Collect PUF when stack VOC concentration } \\
\text { has reached peak/steady state }\end{array}$ & near 296-C-006 stack & $25 \mathrm{~L}$ & $₫ 5 \mathrm{~L} / \mathrm{min}$ \\
\hline 410 & $\begin{array}{l}\text { Collect SUMMA when stack VOC } \\
\text { concentration has reached peak/steady state }\end{array}$ & $\begin{array}{l}\text { downwind at the } \\
\text { fresh air boundary }\end{array}$ & $6 \mathrm{~L}$ & $1 \mathrm{~min}$ \\
\hline
\end{tabular}

Note:

'CPO and SAS may adjust sample durations and flow rates as necessary to collect the samples. 
HNF-4212, Rev. 0

Table 2-4. Vapor Sampling Summary.

\begin{tabular}{|c|c|c|c|c|}
\hline Sample location & Sample time & $\begin{array}{l}\text { Sample } \\
\text { type: }\end{array}$ & Sample container & $\begin{array}{l}\text { Number of } \\
\text { samples }\end{array}$ \\
\hline \multirow[t]{3}{*}{---} & \multirow[t]{3}{*}{ Baseline } & \multirow[t]{3}{*}{ Trip blank } & TST & 1 \\
\hline & & & STT & 1 \\
\hline & & & PUF & 1 \\
\hline \multirow[t]{3}{*}{---} & \multirow[t]{3}{*}{ Baseline } & \multirow[t]{3}{*}{ Field blank } & TST & 1 \\
\hline & & & STT & 1 \\
\hline & & & PUF & 1 \\
\hline \multirow[t]{10}{*}{ 296-C-006 stack } & \multirow[t]{5}{*}{ Baseline } & \multirow[t]{5}{*}{ Stack vapor } & SUMMA & 1 \\
\hline & & & TST & 1 \\
\hline & & & STT & 1 \\
\hline & & & PUF & 1 \\
\hline & & & Particulate filter assembly & 1 \\
\hline & \multirow[t]{5}{*}{ During sluicing } & \multirow[t]{5}{*}{ Stack vapor } & SUMMA & up to 15 \\
\hline & & & TST & up to 15 \\
\hline & & & STT & up to 15 \\
\hline & & & PUF & up to 15 \\
\hline & & & Particulate filter assembly & up to 15 \\
\hline \multirow{4}{*}{$\begin{array}{l}\text { Breathing Area, } \\
\text { C Tank Farm }\end{array}$} & \multirow[t]{4}{*}{ Baseline } & \multirow{4}{*}{$\begin{array}{l}\text { Ambient } \\
\text { air }\end{array}$} & SUMMA & 2 \\
\hline & & & TST & 1 \\
\hline & & & STT & 1 \\
\hline & & & PUF & 1 \\
\hline \multirow{4}{*}{$\begin{array}{l}\text { Breathing Area, } \\
\text { C Tank Farm }\end{array}$} & \multirow[t]{4}{*}{ During sluicing } & \multirow{4}{*}{$\begin{array}{l}\text { Ambient } \\
\text { air }\end{array}$} & SUMMA & 2 \\
\hline & & & TST & 1 \\
\hline & & & STT & 1 \\
\hline & & & PUF & 1 \\
\hline
\end{tabular}

Notes:

baseline $=$ prior to pump startup

Each particulate filter assembly contains two particulate filters mounted in series. 


\section{HNF-4212, Rev. 0}

\subsection{RADIATION SCREENING}

Surveys using particulate filter samples from the 296-C-006 ventilation system will be performed periodically during the process test to allow the samples to obtain a radiological release and ensure that the samples meet the SAS laboratory acceptance criteria. The particulate filter samples will be collected by SAS and sent to WSCF for analysis of both the upstream and downstream particulate filters in each particulate filter assembly. Analytical procedures are specified in Section 3.0. If the limits specified in Section 3.0 are exceeded the survey samples will be recounted every few days until the activity drops below the limits, allowing release of the samples. The results from the radiation screening shall be submitted to SAS and the Process Engineering point of contact for vapor sampling for evaluation. Tritium trap samples were collected and analyzed during phase I of the process test in December 1998. The results of these earlier tritium trap samples were adequate to release the phase II process test samples with regard to potential tritium contamination. Therefore, no tritium trap samples will be obtained during phase III of the process test. 
HNF-4212, Rev. 0

\subsection{LABORATORY ANALYSIS REQUIREMENTS}

The responsibilities of the analytical laboratories are given in this section. Additional quality control and deliverable requirements are given in Sections 4.0 and 7.0.

Vapor samples shall be analyzed by SAS in accordance with Table 3-1. Sorbent tube trains shall be analyzed for ammonia and oxides of nitrogen (nitric oxide and nitrogen dioxide). SUMMA canisters shall be analyzed for total non-methane hydrocarbons, hydrogen, nitrous oxide, methane, carbon monoxide, carbon dioxide, and selected organic analytes. TSTs shall be analyzed for selected organic analytes. PUF samples shall be analyzed for tri-butyl phosphate (TBP) and di-butyl phosphate (DBP).

Required analytes are those listed in Tables 3-2, 3-3, and 3-4. Quantitation limit goals for the Class A and B toxic air pollutants in these tables are documented in Mulkey (1995). In addition to the compounds listed, a determination is to be made for all other peaks that are at least 10 percent of the nearest internal standard. If possible, peaks smaller than 10 percent of the nearest internal standard should also be identified. All major constituents in the sample should be identified.

It is expected that not all samples will be analyzed. It is expected that, at a minimum, the laboratory will be directed to analyze all trip blanks, field blanks, breathing area samples, baseline samples, and at least three sets of samples from 296-C-006 stack. Prior to the receipt of the samples at the laboratory, the Process Engineering point of contact for vapor sampling shall provide SAS with a list identifying the stack samples to be analyzed.

Particulate filter samples shall be analyzed at the WSCF as discussed in Section 2.3 and in accordance with Table 3-1.

If any requested analyses cannot be performed, the Process Engineering point of contact for vapor sampling shall be notified. 


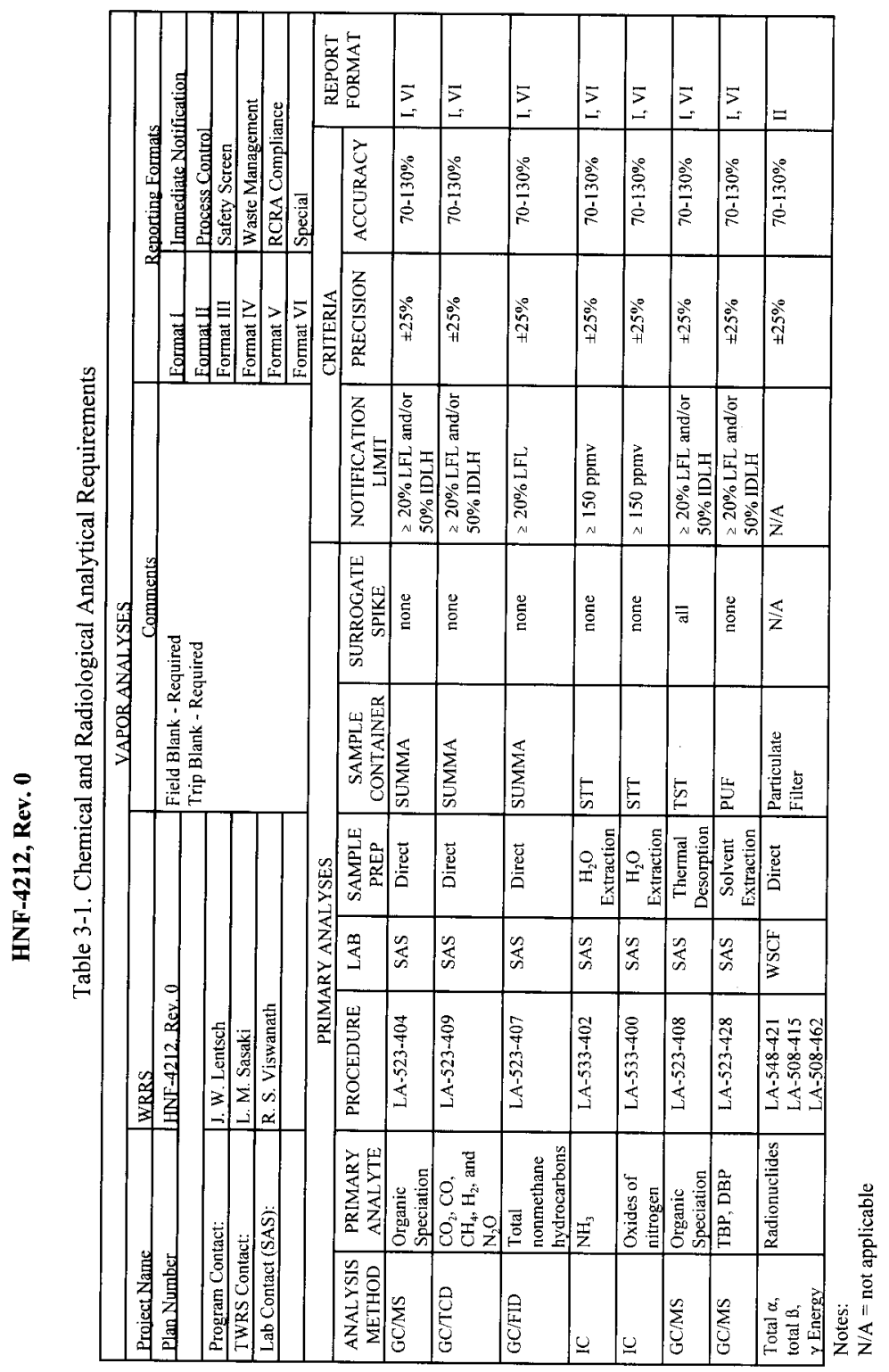


HNF-4212, Rev. 0

Table 3-2. Required Analytes: Regulatory

\begin{tabular}{|l|r|r|r|r|r|}
\hline \multicolumn{1}{|c|}{ Analyte } & CAS & LFL & LFL & IDLH & WLH \\
& Number & (ppmv) & (ppmv) & (ppmv) & (ppmv) \\
\hline 1,3 Butadiene & $106-99-0$ & 20,000 & 4,000 & 2,000 & 1000 \\
\hline Chloroform & $67-66-3$ & N/A & N/A & 500 & 250 \\
\hline 1, 4 Dioxane & $123-91-1$ & 20,000 & 4,000 & 500 & 250 \\
\hline Benzene & $71-43-2$ & 12,000 & 2,400 & 500 & 250 \\
\hline Carbon tetrachloride & $56-23-5$ & N/A & N/A & 200 & 100 \\
\hline Dichloromethane (Methylene chloride) & $75-09-2$ & 130,000 & 26,000 & 2,300 & 1,150 \\
\hline Acetaldehyde & $75-07-1$ & 40,000 & 8,000 & 2,000 & 1,000 \\
\hline Perchloroethylene (Tetrachloroethylene) & $127-18-4$ & N/A & N/A & 150 & 75 \\
\hline Ammonia & $7664-41-7$ & 150,000 & 30,000 & 300 & 150 \\
\hline Tributyl phosphate & $126-73-8$ & N/A & N/A & 30 & 15 \\
\hline Dibutyl phosphate & $107-66-4$ & N/A & N/A & 30 & 15 \\
\hline Hexane & $110-54-3$ & 12,000 & 2,400 & 1,100 & 550 \\
\hline Nitric oxide & $10102-43-9$ & N/A & N/A & 100 & 50 \\
\hline Acetone & $67-64-1$ & 25,000 & 5,000 & 2,500 & 1,250 \\
\hline n-Butanol & $71-36-3$ & 14,000 & 2,800 & 1,400 & 700 \\
\hline Nitrous oxide & $10024-97-2$ & N/A & N/A & N/A & N/A \\
\hline 3-Heptanone (Ethyl butyl ketone) & $106-35-4$ & N/A & N/A & 1,000 & 500 \\
\hline Nonane & $111-84-2$ & 8,500 & 1,700 & N/A & N/A \\
\hline 4-Heptanone (Dipropyl ketone) & $123-19-3$ & N/A & N/A & N/A & N/A \\
\hline n-Heptane & $142-82-5$ & 10,500 & 2,100 & 750 & 375 \\
\hline TNMHC & N/A & N/A & N/A & N/A & N/A \\
\hline
\end{tabular}

Note:

$\mathrm{N} / \mathrm{A}=$ not applicable or not available 
Table 3-3. Required Analytes: Program Requested Analytes/Selected Compounds Observed in Tank 241-C-106 Vapor or Waste Samples

\begin{tabular}{|l|r|r|r|r|r|}
\hline Analyte & $\begin{array}{r}\text { CAS } \\
\text { Number }\end{array}$ & $\begin{array}{r}\text { LFL } \\
\text { (ppmv) }\end{array}$ & $\begin{array}{r}\text { LFL } \\
\text { (ppmv) }\end{array}$ & $\begin{array}{r}\text { IDLH } \\
\text { (ppmv) }\end{array}$ & $\begin{array}{c}50 \% \\
\text { IDLH } \\
\text { (ppmv) }\end{array}$ \\
\hline Methane & $74-82-8$ & 50,000 & 10,000 & N/A & N/A \\
\hline Ethane & $74-84-0$ & 30,000 & 6,000 & N/A & N/A \\
\hline Ethylene & $74-85-1$ & 30,000 & 6,000 & N/A & N/A \\
\hline 1-Hexene & $592-41-6$ & N/A & N/A & N/A & N/A \\
\hline Hexane & $110-54-3$ & 12,000 & 2,400 & 1,100 & 550 \\
\hline 1-Heptene & $582-76-7$ & N/A & N/A & N/A & N/A \\
\hline 3-Heptene, (E)- & $14686-14-7$ & N/A & N/A & N/A & N/A \\
\hline Heptane & $142-82-5$ & 10,500 & 2,100 & 750 & 375 \\
\hline 2-Heptene & $592-77-8$ & N/A & N/A & N/A & N/A \\
\hline 3-Heptene & $292-78-9$ & N/A & N/A & N/A & N/A \\
\hline 2,4-Dimethyl-2,3-pentadiene & $1000-87-9$ & N/A & N/A & N/A & N/A \\
\hline 2-Heptyne & $1119-65-9$ & N/A & N/A & N/A & N/A \\
\hline 3-Methyl-heptane & $589-81-1$ & N/A & N/A & N/A & N/A \\
\hline 3-Methylene-heptane & $1632-16-2$ & N/A & N/A & N/A & N/A \\
\hline 3-Methyl-3-heptene & $7300-03-0$ & N/A & N/A & N/A & N/A \\
\hline 3-Ethyl-4-methyl-4-pentene & $61847-80-1$ & N/A & N/A & N/A & N/A \\
\hline 2-Octene & $111-67-1$ & N/A & N/A & N/A & N/A \\
\hline 4-Heptanone & $123-19-3$ & N/A & N/A & N/D & N/D \\
\hline 3-Heptanone & $106-35-4$ & N/A & N/A & 1,000 & 500 \\
\hline Nonane & $111-84-2$ & 8,500 & 1,700 & N/A & N/A \\
\hline Decane & $124-18-5$ & 7,500 & 1,500 & N/A & N/A \\
\hline Decahydro-naphthalene & $91-17-8$ & 7,000 & 1,400 & N/A & N/A \\
\hline Undecane & $1120-21-4$ & N/A & N/A & N/A & N/A \\
\hline Dodecane & $112-40-3$ & 6,000 & 1,200 & N/A & N/A \\
\hline Tridecane & $629-50-5$ & N/A & N/A & N/A & N/A \\
\hline Tetradecane & $629-59-4$ & N/A & N/A & N/A & N/A \\
\hline Pentadecane & $629-62-9$ & N/A & N/A & N/A & N/A \\
\hline Nene & & & & & \\
\hline
\end{tabular}

Note:

N/A = not applicable or not available 
HNF-4212, Rev, 0

Table 3-4. Required Analytes: Target Analytes (2 sheets).

\begin{tabular}{|c|c|c|c|c|c|}
\hline Analyte & $\begin{array}{c}\text { CAS } \\
\text { Number } \\
\end{array}$ & $\begin{array}{l}\text { LFL } \\
\text { (ppmv) }\end{array}$ & $\begin{array}{c}20 \% \\
\text { LFL } \\
\text { (ppmy) }\end{array}$ & $\begin{array}{l}\text { IDIH } \\
\text { (pprnv) }\end{array}$ & $\begin{array}{c}50 \% \\
\text { IDLH } \\
\text { (ppmv) }\end{array}$ \\
\hline Ammonia, $\mathrm{NH}_{3}$ & $7664-41-7$ & 150,000 & 30,000 & 300 & 150 \\
\hline Nitric oxide, NO & $10102-43-9$ & N/A & $\mathrm{N} / \mathrm{A}$ & 100 & 50 \\
\hline Nitrogen dioxide, $\mathrm{NO}_{2}$ & $10102-44-0$ & N/A & N/A & 20 & 10 \\
\hline Methane, $\mathrm{CH}_{4}$ & $74-82-8$ & 50,000 & 10,000 & $N / A$ & $\mathrm{~N} / \mathrm{A}$ \\
\hline Carbon dioxide, $\mathrm{CO}_{2}$ & $124-38-9$ & $\mathrm{~N} / \mathrm{A}$ & $\mathrm{N} / \mathrm{A}$ & 40,000 & 20,000 \\
\hline Carbon monoxide, $\mathrm{CO}$ & $630-08-0$ & 125,000 & 25,000 & 1,200 & 600 \\
\hline Nitrous oxide, $\mathrm{N}_{2} \mathrm{O}$ & $10024-97-2$ & N/A & N/A & N/A & $\mathrm{N} / \mathrm{A}$ \\
\hline Hydrogen, $\mathrm{H}_{2}$ & $1333-74-0$ & 40,000 & 8,000 & N/A & $\mathrm{N} / \mathrm{A}$ \\
\hline Acetaldehyde & $75-07-1$ & 40,000 & 8,000 & 2,000 & 1,000 \\
\hline Dichlorodifluoromethane (Freon 12) & $75-71-8$ & $\mathrm{~N} / \mathrm{A}$ & $\mathrm{N} / \mathrm{A}$ & 15,000 & 7,500 \\
\hline Methyl chloride & $74-87-3$ & 81,000 & 16,200 & 2,000 & 1,000 \\
\hline n-Butane & $106-97-8$ & 18,000 & 3,600 & N/A & $\mathrm{N} / \mathrm{A}$ \\
\hline Ethyl chloride & $75-00-3$ & 38,000 & 7,600 & 3,800 & 1,900 \\
\hline Ethanol & $64-17-5$ & 33,000 & 6,600 & 3,300 & 1,650 \\
\hline Trichlorofluoromethane (Freon 11) & $75-69-4$ & N/A & $\mathrm{N} / \mathrm{A}$ & 2,000 & 1,000 \\
\hline Ethanenitrile (acetonitrile) & $75-05-8$ & 30,000 & 6,000 & 500 & 250 \\
\hline Propanone (acetone) & $67-64-1$ & 25,000 & 5,000 & 2,500 & 1,250 \\
\hline Furan & $110-00-9$ & N/A & $\mathrm{N} / \mathrm{A}$ & $\mathrm{N} / \mathrm{A}$ & $\mathrm{N} / \mathrm{A}$ \\
\hline n-Pentane & $109-66-0$ & 14,000 & 2,800 & 1,500 & 750 \\
\hline 2-Propanol & $67-63-0$ & 20,000 & 4,000 & 2,000 & 1,000 \\
\hline Dichloromethane (methylene chloride) & $75-09-2$ & 130,000 & 26,000 & 2,300 & 1,150 \\
\hline 1-Propanol & $71-23-8$ & 22,000 & 4,400 & 800 & 400 \\
\hline 2-Methyl pentane & $107-83-5$ & $\mathrm{~N} / \mathrm{A}$ & $\mathrm{N} / \mathrm{A}$ & N/A & N/A \\
\hline Propanenitrile & $107-12-0$ & 31,000 & 6,200 & N/A & $\mathrm{N} / \mathrm{A}$ \\
\hline Butanal & $123-72-8$ & 14,000 & 2,800 & 1,400 & 700 \\
\hline 1-Hexene & $592-41-6$ & N/A & $\mathrm{N} / \mathrm{A}$ & $\mathrm{N} / \mathrm{A}$ & N/A \\
\hline 2-Butanone & $78-93-3$ & 14,000 & 2,800 & 3,000 & 1,500 \\
\hline n-Hexane & $110-54-3$ & 12,000 & 2,400 & 1,100 & 550 \\
\hline Trichloromethane (chloroform) & $67-66-3$ & $\mathrm{~N} / \mathrm{A}$ & N/A & 500 & 250 \\
\hline Tetrahydrofuran & $109-99-9$ & 20,000 & 4,000 & 2,000 & 1,000 \\
\hline 1-Butanol & $71-36-3$ & 14,000 & 2,800 & 1,400 & 700 \\
\hline Benzene & $71-43-2$ & 12,000 & 2,400 & 500 & 250 \\
\hline $\begin{array}{l}\text { Tetrachloromethane (carbon } \\
\text { tetrachloride) }\end{array}$ & $56-23-5$ & $\mathrm{~N} / \mathrm{A}$ & N/A & 200 & 100 \\
\hline 1-Butanenitrile & $109-74-0$ & 16,500 & 3,300 & N/A & N/A \\
\hline 3-Methyl hexane & $589-34-4$ & $\mathrm{~N} / \mathrm{A}$ & $\mathrm{N} / \mathrm{A}$ & $\mathrm{N} / \mathrm{A}$ & $\mathrm{N} / \mathrm{A}$ \\
\hline 2-Pentanone & $107-87-9$ & 15,000 & 3,000 & 1,500 & 750 \\
\hline
\end{tabular}


HNF-4212, Rev. 0

Table 3-4. Required Analytes: Target Analytes (2 sheets).

\begin{tabular}{|c|c|c|c|c|c|}
\hline Analyte & $\begin{array}{c}\text { CAS } \\
\text { Number }\end{array}$ & $\begin{array}{c}\text { LFL } \\
\text { (ppmv) }\end{array}$ & $\begin{array}{c}20 \% \\
\text { LFI } \\
\text { (ppmv) }\end{array}$ & $\begin{array}{l}\text { IDLH } \\
\text { (ppmv) }\end{array}$ & $\begin{array}{l}50 \% \\
\text { IDLH } \\
\text { (ppmv) }\end{array}$ \\
\hline n-Heptane & $142-82-5$ & 10,500 & 2,100 & 750 & 375 \\
\hline 1,4-Dioxane & $123-91-1$ & 20,000 & 4,000 & 500 & 250 \\
\hline 4-Methyl-2-pentanone (hexone) & $108-10-1$ & 12,000 & 2,400 & 500 & 250 \\
\hline Toluene & $108-88-3$ & 11,000 & 2,200 & 500 & 250 \\
\hline 2-Hexanone & $591-78-6$ & $\mathrm{~N} / \mathrm{A}$ & $\mathrm{N} / \mathrm{A}$ & 150 & 75 \\
\hline n-Octane & $111-65-9$ & 9,500 & 1,900 & 1,000 & 500 \\
\hline Tetrachloroethylene & $127-18-4$ & $\mathrm{~N} / \mathrm{A}$ & $\mathrm{N} / \mathrm{A}$ & 150 & 75 \\
\hline Chlorobenzene & $108-90-7$ & 13,000 & 2,600 & 1,000 & 500 \\
\hline Ethylbenzene & $100-41-4$ & 8,000 & 1,600 & 800 & 400 \\
\hline $\mathrm{m}$, p-Xylene & $106-42-3$ & 11,000 & 2,200 & 900 & 450 \\
\hline 3-Heptanone & $106-35-4$ & $\mathrm{~N} / \mathrm{A}$ & N/A & 1,000 & 500 \\
\hline 2-Heptanone & $110-43-0$ & 11,000 & 2,200 & 800 & 400 \\
\hline Cyclohexanone & $108-94-1$ & 11,000 & 2,200 & 700 & 350 \\
\hline Styrene & $100-42-5$ & 9,000 & 1,800 & 700 & 350 \\
\hline n-Nonane & $111-84-2$ & 8,500 & 1,700 & $\mathrm{~N} / \mathrm{A}$ & $\mathrm{N} / \mathrm{A}$ \\
\hline o-Xylene & $95-47-6$ & 9,000 & 1,800 & 900 & 450 \\
\hline 1,1,2,2-Tetrachloroethane & $79-34-5$ & $\mathrm{~N} / \mathrm{A}$ & N/A & 100 & 50 \\
\hline 2-Octanone & $111-13-7$ & $\mathrm{~N} / \mathrm{A}$ & $\mathrm{N} / \mathrm{A}$ & $\mathrm{N} / \mathrm{A}$ & $\mathrm{N} / \mathrm{A}$ \\
\hline n-Decane & $124-18-5$ & 7,500 & 1,500 & $\mathrm{~N} / \mathrm{A}$ & N/A \\
\hline 1,2,4-Trimethylbenzene & $95-63-6$ & 9,000 & 1,800 & $\mathrm{~N} / \mathrm{A}$ & N/A \\
\hline 1-heptene & $592-76-7$ & 10,000 & 2,000 & N/A & N/A \\
\hline trans-2-heptene & 14686-13-6 & N/A & N/A & $\mathrm{N} / \mathrm{A}$ & $\mathrm{N} / \mathrm{A}$ \\
\hline cis-2-heptene & $6443-92-1$ & $\mathrm{~N} / \mathrm{A}$ & N/A & $\mathrm{N} / \mathrm{A}$ & N/A \\
\hline trans-3-heptene & 14686-14-7 & $\mathrm{N} / \mathrm{A}$ & $\mathrm{N} / \mathrm{A}$ & $\mathrm{N} / \mathrm{A}$ & $\mathrm{N} / \mathrm{A}$ \\
\hline cis-3-heptene & $7642-10-6$ & N/A & $\mathrm{N} / \mathrm{A}$ & $\mathrm{N} / \mathrm{A}$ & $\mathrm{N} / \mathrm{A}$ \\
\hline
\end{tabular}

Note:

$\mathrm{N} / \mathrm{A}=$ not applicable or not available 


\subsection{QUALITY ASSURANCE AND QUALITY CONTROL}

Vapor sampling and analysis shall be performed in accordance with approved quality assurance (QA) plans. These plans are required to meet the Hanford Analytical Services Quality Assurance Requirements Document (HASQARD) (DOE 1998) requirements. Validation of this compliance shall be verified either by a HASQARD assessment stating their quality program satisfactorily meets the appropriate requirements, or the quality program plan and applicable procedures will be submitted and approved prior to work performance on sampling or analytical work. Quality requirements for conducting Characterization Project sampling and analysis are described in Tank Waste Remediation System Characterization Project, Quality Policies (Board 1998) and this sampling and analysis plan. Characterization Project sampling and analysis shall be conducted in conformance with these QA requirements.

Processes, services, activities, and conditions adverse to quality which do not conform to requirements specified in this sampling and analysis plan or references herein shall be controlled to prevent inadvertent use. Nonconforming sampling and analysis processes shall be identified, controlled, reported, and dispositioned as required by PHMC (1998).

\subsection{LABORATORY OPERATIONS}

Analytical procedures that are to be used for this sample and analysis plan shall be written and approved prior to the performance of analytical work within the laboratory. Analytical QC requirements are identified in Table $3-1$. The laboratory shall also use calibration and calibration check standards appropriate for the analytical instrumentation being used (see DOE [1998] for definitions of QC samples and standards). The criteria presented are goals for demonstrating reliable method performance. It is understood that the laboratory will follow its internal QC system for required actions whenever QC failures occur. If sample QC failures occur or if all analyses cannot be performed (e.g., insufficient sample), analysts shall consult with supervisors/customers to determine the proper action. The laboratory should provide a suggested course of action at that time. All sample QC failures and limitations on the associated data shall be discussed in the narrative of the data report. Proper notification of all data not meeting QC requirements shall be included with the data.

\subsection{SAMPLE COLLECTION}

Sampling shall be performed in accordance with approved procedures and work plans included within the job control system. All data sheets and log entries completed during the performance of sampling shall be copied and included within the job control system package.

Each sample identification number shall have the following format:

\section{VLXXX-YYY-ZZZZ}




\section{HNF-4212, Rev. 0}

where,

$\mathbf{V}$ indicates a vapor sample,

$\mathbf{L}=$ a letter code identifying the organization that prepared the sample container/sample media $\mathrm{S}=\mathrm{SAS}$,

$\mathbf{X X X}=$ a three-digit/letter code identifying the sample location $006=296-\mathrm{C}-006$ stack

$\mathrm{AMB}=$ ambient air samples at $\mathrm{C}$ tank farm

YYY = a three-digit sample code found in Tables 2-2 and 2-3

$\mathbf{Z Z Z Z}=$ a special lab-assigned code.

\subsection{SAMPLE CUSTODY}

Chain-of-custody will be carefully maintained to assure sample control at all times. 


\section{HNF-4212, Rev. 0}

\subsection{EXCEPTIONS, CLARIFICATIONS, AND ASSUMPTIONS}

\section{Trip Blanks and Field Blanks}

Trip Blanks are sampling devices prepared and handled in the same manner as samples, except that they are never opened in the field. Field Blanks are sampling devices prepared and handled in the same manner as the samples, but no tank gases are drawn through them. 
HNF-4212, Rev. 0

\subsection{ORGANIZATION}

The organization and responsibility of key personnel involved with these tank characterization projects are listed in Table 6-1.

Table 6-1. Project Key Personnel

\begin{tabular}{|c|c|c|}
\hline Responsibility & Organization & Individual \\
\hline $\begin{array}{l}\text { Data Assessment and } \\
\text { Interpretation manager }\end{array}$ & $\begin{array}{l}\text { TWRS Process Engineering } \\
\text { (LMHC) }\end{array}$ & K. M. Hall, 376-5029 \\
\hline $\begin{array}{l}\text { Process Engineering point of } \\
\text { contact for vapor sampling }\end{array}$ & $\begin{array}{l}\text { TWRS Process Engineering } \\
\text { (LMHC) }\end{array}$ & L. M. Sasaki, 373-1027 \\
\hline $\begin{array}{l}\text { Waste retrieval sluicing } \\
\text { system operations manager }\end{array}$ & $\begin{array}{l}\text { Retrieval Operations Program } \\
(\mathrm{NHC})\end{array}$ & J. W. Lentsch, 373-5252 \\
\hline $\begin{array}{l}\text { Waste retrieval sluicing } \\
\text { system technical contact }\end{array}$ & $\begin{array}{l}\text { TWRS Process Engineering, } \\
\text { (LMHC) }\end{array}$ & K. G. Carothers, 373-4556 \\
\hline $\begin{array}{l}\text { Vapor sampling cognizant } \\
\text { engineer }\end{array}$ & $\begin{array}{l}\text { Characterization Field } \\
\text { Engineering (LMHC) }\end{array}$ & D. D. Wanner, 373-3297 \\
\hline $\begin{array}{l}\text { SAS vapor sampling and } \\
\text { analysis project manager }\end{array}$ & $\begin{array}{l}\text { Special Analytical Support } \\
\text { (NHC) }\end{array}$ & L. L. Lockrem, 373-4771 \\
\hline $\begin{array}{l}\text { SAS vapor sampling and } \\
\text { analysis technical contact }\end{array}$ & $\begin{array}{l}\text { Special Analytical Support } \\
(\mathrm{NHC})\end{array}$ & R. S. Viswanath, 376-9223 \\
\hline $\begin{array}{l}\text { Industrial Hygiene and Safety } \\
\text { points of contact }\end{array}$ & $\begin{array}{l}\text { Tank Farm Facilities } \\
\text { Operations, Fieid Safety } \\
\text { Services (LMHC) }\end{array}$ & $\begin{array}{l}\text { K. M. Bowen, 372-3667 } \\
\text { N. K. Butler, } 376-5795\end{array}$ \\
\hline $\begin{array}{l}\text { Double-Shell Tank Farm } \\
\text { point of contact }\end{array}$ & Tank Farm Operations & $\begin{array}{l}\text { Double-Shell Tank Farm } \\
\text { Operations shift manager, } \\
373-2689\end{array}$ \\
\hline $\begin{array}{l}\text { Senior Supervisory Watch, } \\
\text { MO-2.11 }\end{array}$ & $\begin{array}{l}\text { C-106 Sluicing Operations } \\
(\mathrm{LMHC})\end{array}$ & G. N. Hanson, 376-2182 \\
\hline $\begin{array}{l}\text { Test Director, Waste } \\
\text { Retrieval Sluicing System } \\
\text { Process Test }\end{array}$ & $\begin{array}{l}\text { C-106 Sluicing Operations } \\
\text { (LMHC) }\end{array}$ & K. J. Anderson, 373-6039 \\
\hline $\begin{array}{l}\text { Environmental point of } \\
\text { contact }\end{array}$ & $\begin{array}{l}\text { Environmental } \\
\text { Permits/Policy (LMHC) }\end{array}$ & G. M. Crummel, 373-5175 \\
\hline
\end{tabular}




\subsection{DELIVERABLES}

Sampling and analytical results shall be reported as Format VI reports. Any analyte exceeding the notification limit prescribed in Table 3-1 shall also be reported as a Format I report. In addition, Format II reports shall be provided by SAS, CPO, and WSCF as described in Section 7.2 .

\subsection{FORMAT I REPORTING}

Table 3-1 contains the notification limits for specific analytes. Analytes that exceed notification limits shall be reported by the Project Manager or delegate by calling the Double-Shell Tank Farms Operations shift manager as soon as the data are obtained and reviewed by the responsible scientist. This verbal notification must be followed within one hour by electronic notification to the Double-Shell Tank Farms Operations shift manager, the Industrial Hygiene and Safety point of contact, the TWRS Process Engineering Data Assessment and Interpretation manager, the waste retrieval sluicing system point of contact and the Process Engineering point of contact for vapor sampling. A further review of the data, including quality control results and additional analyses for verification purposes may be contracted with the performing laboratory by either a revision to this sampling and analysis plan or by a letter.

\subsection{FORMAT II REPORTING}

WSCF shall provide the results of radiological analyses using the standard WSCF analytical laboratory report format. The results shall be faxed to SAS and the Process Engineering vapor sampling point of contact within 48 hours of receipt of the samples and followed by transmittal of a copy of the results via plant mail.

CPO shall provide information on its sampling activities (copies of sampling data sheets, J-5 forms, chain of custody forms, and other pertinent documentation) to SAS and the Process Engineering vapor sampling point of contact. The information provided shall include: the sample collection sequence and volumes, start and stop times for the collection of each sample, the VOC reading at the start of the collection of each sample, and any anomalous sampling conditions. This information shall be provided within 48 hours of the collection of the last set of samples.

The SAS sampling team shall provide information on its sampling activities to the Process Engineering vapor sampling point of contact. The information provided shall include: the sample collection sequence and volumes, start and stop times for the collection of each sample, the VOC reading at the start of the collection of each sample, verification of trip and field blank use, and any anomalous sampling conditions. This information shall be provided within 48 hours of the collection of the last set of samples. 


\subsection{FORMAT VI REPORTING}

The Format VI report shall consist of two deliverables, preliminary analytical results and a final data package.

Preliminary sampling and analytical data shall be delivered within two weeks of the receipt of the samples at the laboratory. The preliminary data shall consist of, at a minimum, data tables reporting sample collection data, particulate filter analysis results, and the results of each analysis performed by the analytical laboratory. The following individuals shall be on distribution for the preliminary results: J. W. Bailey, N. K. Butler, K. G. Carothers, G. M. Crummel, J. W. Lentsch, C. H. Mulkey, R. L. Powers, and L. M. Sasaki.

A data package shall be issued as a supporting document within seven weeks of the receipt of the samples at the laboratory. The data package shall contain the elements listed in Table 7-1. The following individuals shall be on distribution for the entire data package: R. A. Bechtold, G. M. Crummel, and L. M. Sasaki. The following individuals require only the engineering data transmittal: J. W. Bailey, K. G. Carothers, J. W. Lentsch, N. K. Butler, R. L. Powers, and C. A. Simonen.

In addition to the data package, an electronic version of the analytical results shall be provided to the Tank Vapor Database representative within 4 calendar days from the day that the final data package is issued. The data must be available to the Washington State Department of Ecology within 7 calendar days of release of the data package. The electronic version shall be in the standard electronic format specified in Bobrowski and Simonen (1999).

Table 7-1. Data Package Required Elements (2 sheets).'

\begin{tabular}{|l|}
\hline \multicolumn{1}{|c|}{ Prefatory Elements } \\
\hline Executive summary \\
\hline Table of contents \\
\hline List of abbreviations and acronyms \\
\hline Quality assurance data package review results \\
\hline Non-conformance reports \\
\hline
\end{tabular}


HNF-4212, Rev. 0

Table 7-1. Data Package Required Elements (2 sheets). ${ }^{1}$

Sampling Elements

Sampling case narrative

Sample summary and event chronology

Sampling procedures table

Sampling logbook table

Field data

Radiation screening results

Chain of custody forms

Analysis Elements

Analytical case narrative

Analytical procedures table

Data qualifier flag translation table

Target analytes concentration table

Tentatively identified compound concentration table

Laboratory blank summary

Field blank summary

Trip blank summary

Mass spectrometer instrument tune report

Target analyte initial calibration table

Internal standards area counts table

Laboratory control sample results table

Surrogate compounds results table

Quantitation reports

Chromatograms

Mass spectra of reported tentatively identified compounds

Note:

${ }^{1}$ Include all elements as applicable. 
HNF-4212, Rev. 0

\subsection{CHANGE CONTROL}

Under certain circumstances, it may become necessary for the performing laboratory to make decisions concerning a sample without review of the data by the customer or the Characterization Project. All significant changes shall be documented by TWRS Process Engineering via an engineering change notice to this sampling and analysis plan or by a letter. All changes shall also be clearly documented in the final data report. Insignificant changes may be made by placing a notation in the permanent record (i.e., note change in log book or memo to file). Significance is determined by the Process Engineering point of contact for vapor sampling.

At the request of the Retrieval Operations Project, additional analysis of sample material from this characterization project shall be performed following a revision of this sampling and analysis plan or issuance of a letter. 


\subsection{REFERENCES}

Board, D. C., 1998, Tank Waste Remediation System, Characterization Project, Quality Policies, HNF-SD-WM-QAPP-025, Rev. 4, Lockheed Martin Hanford Corp. for Fluor Daniel Hanford, Inc., Richland, Washington.

Bobrowski, S. F., and C. A. Simonen, 1999, Standard Electronic Format Specification for Tank Vapor Data MSEXCEL Spreadsheets: Version 1.0, HNF-3815, Rev. 0, prepared by Pacific Northwest National Laboratory for Lockheed Martin Hanford Corp, Richland, Washington.

DOE, 1998, Hanford Analytical Services Quality Assurance Requirements Document, DOE/RL96-68, Rev. 2, U.S. Department of Energy, Richland Field Office, Richland, Washington.

Jones, J. M., 1999, Waste Retrieval Sluicing System Emissions Collection Phase III, PTP-320001, Rev. A-3, Lockheed Martin Hanford Corp. for Fluor Daniel Hanford, Inc., Richland, Washington.

Mulkey, C. H., and K. D. Markillie, 1995, Data Quality Objectives for Regulatory Requirements for Hazardous and Radioactive Air Emissions Sampling and Analysis, WHC-SD-WM-DQO021, Rev. 0, Westinghouse Hanford Company, Richland, Washington.

Peterson, K. A., 1998, Request for Ecology Concurrence on Draft Strategy/Path Forward to Address Concerns Regarding Organic Emissions from C-106 Sluicing Activities, (Meeting Minutes, December 3), Fluor Daniel Hanford, Inc., Richland, Washington.

PHMC, 1998, Nonconforming Item Reporting and Control, HNF-PRO-298, Rev. 1, Fluor Daniel Hanford, Inc. Richland, Washington.

Powers, R. L., 1999, Process Test Plan, Phase III: Waste Retrieval Sluicing System Emissions Collection, HNF-4034, Rev. 1, Lockheed Martin Hanford Corp. for Fluor Daniel Hanford, Inc., Richland, Washington. 


\section{DISTRIBUTION SHEET}

\begin{tabular}{|c|c|c|c|c|c|}
\hline \multirow{2}{*}{ Do } & \multirow{2}{*}{\multicolumn{3}{|c|}{$\begin{array}{l}\text { From } \\
\text { Data Assessment and } \\
\text { Interpretation }\end{array}$}} & \multicolumn{2}{|l|}{ Page 1 of 2} \\
\hline & & & & \multicolumn{2}{|c|}{$03 / 15 / 99$} \\
\hline \multicolumn{4}{|l|}{ Project Title/Work Order } & \multicolumn{2}{|c|}{ EDT No. EDT-611456 } \\
\hline \multicolumn{4}{|c|}{$\begin{array}{l}\text { HNF-4212, Rev. 0, "Waste Retrieval Sluicing System Vapor } \\
\text { Sampling and Analysis Plan for Evaluation of Organic Emissions, } \\
\text { Process Test Phase III" }\end{array}$} & \multicolumn{2}{|c|}{ ECN No. $\quad \mathrm{N} / \mathrm{A}$} \\
\hline \multicolumn{2}{|l|}{ Name } & $\begin{array}{l}\text { Text } \\
\text { With } \\
\text { All } \\
\text { Attach. }\end{array}$ & Text Only & $\begin{array}{l}\text { Attach./ } \\
\text { Appendix } \\
\text { Only }\end{array}$ & $\begin{array}{c}\mathrm{EDT} / \mathrm{ECN} \\
\text { Only }\end{array}$ \\
\hline
\end{tabular}

U. S. Department of Energy -

Richland Field office

W. AbduT

DOE/RL Reading Room

$\begin{array}{ll}\mathrm{S} 7-54 & \mathrm{X} \\ \mathrm{H} 2-53 & \mathrm{X}\end{array}$

\section{COGEMA}

J. R. Bellomy

L. A. Pingel

R. S. Viswanath

S5-05

S3-90

S3-90

Fluor Daniel Northwest

D. L. Evans

S2-47 $\quad X$

Lockheed Martin Hanford Corp.

K. J. Anderson

D. C. Board

K. M. Bowen

R. G. Brown

R. L. Brown

W. E. Bryan

N. K. Butler

K. G. Carothers

G. M. Crummel

K. M. Hall

G. N. Hanson

T. D. Jarecki

J. A. Johnston

N. W. Kirch

C. H. Mulkey

R. L. Powers

L. M. Sasaki

L. A. Stauffer

D. D. Wanner

T.C.S.R.C.

$\begin{array}{ll}\text { S5-04 } & X \\ \text { S7-07 } & X \\ \text { S5-12 } & X \\ \text { S7-12 } & X \\ \text { S6-14 } & X \\ \text { S5-05 } & X \\ \text { S5-12 } & X \\ \text { R2-11 } & X \\ \text { R1-51 } & X \\ \text { R2-12 } & X \\ \text { S5-07 } & X \\ \text { S7-03 } & X \\ \text { R2-12 } & X \\ \text { R2-11 } & X \\ R 1-51 & X \\ \text { S5-13 } & X \\ \text { R2-12 } & X \\ R 2-11 & X \\ S 7-12 & X \\ R 1-10 & X\end{array}$

Lockheed Martin Services, Inc.

Central Files

B1-07 $X$

Los Alamos Technical Associates

J. M. Jones

S5-13 X 


\section{DISTRIBUTION SHEET}

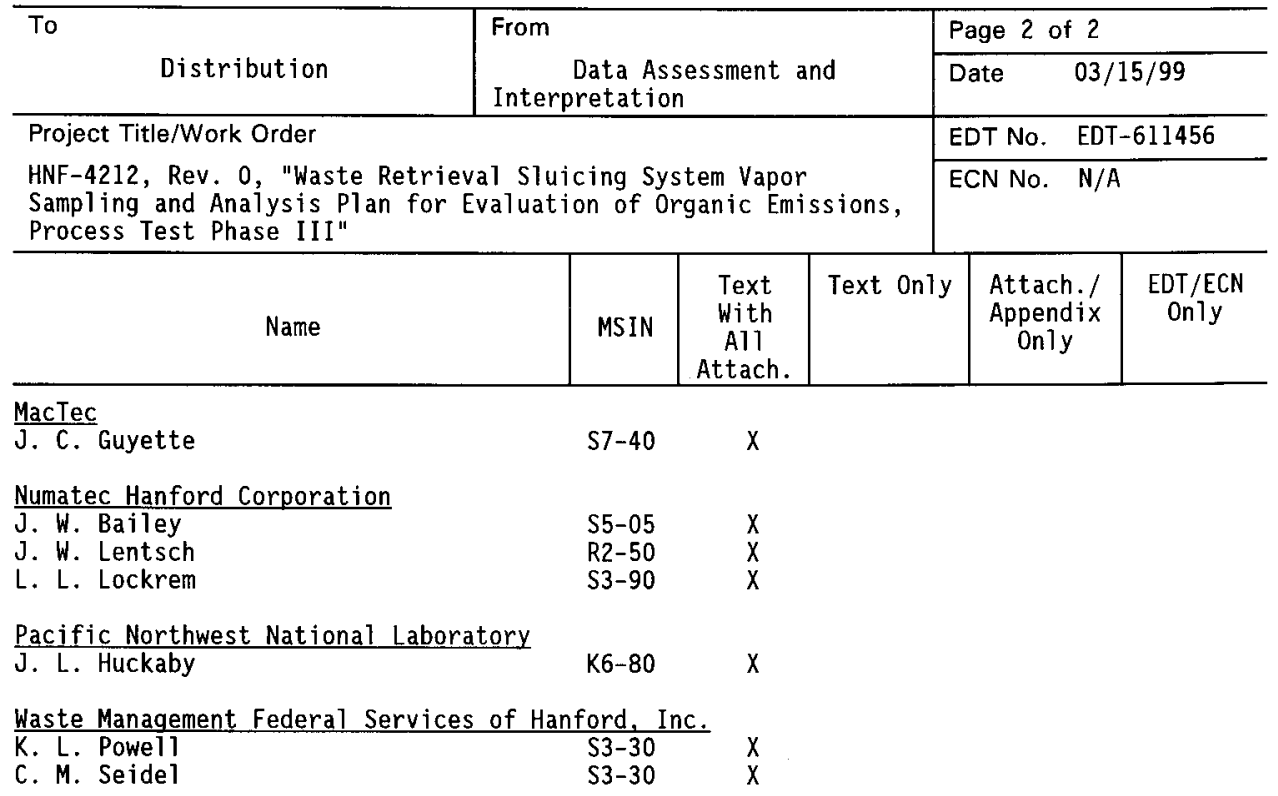

